01

\title{
Рекуррентная процедура построения неизотропных матричных элементов интеграла столкновений нелинейного уравнения Больцмана
}

\author{
(С) И.А. Эндер, ${ }^{2}$ Л.А. Бакалейников, ${ }^{1}$ Е.Ю. Флегонтова, ${ }^{1}$ А.Б. Герасименко \\ ${ }^{1}$ Физико-технический институт им. А.Ф. Иофре РАН, \\ 194021 Санкт-Петербург, Россия \\ ${ }^{2}$ Санкт-Петербургский государственный университет, \\ 199034 Санкт-Петербург, Россия \\ e-mail: fl.xiees@mail.ioffe.ru
}

(Поступило в Редакцию 27 декабря 2016 г.)

Предложен алгоритм последовательного построения неизотропных матричных элементов интеграла столкновений, необходимых для решения нелинейного уравнения Больцмана моментным методом. Стартовыми при этом являются изотропные матричные элементы, считающиеся известными. Алгоритм может быть использован для произвольного закона взаимодействия при любом отношении масс сталкивающихся частиц.

DOI: $10.21883 / J T F .2017 .08 .44718 .2154$

\section{Введение}

Многие современные прикладные и технические проблемы требуют глубокого изучения кинетических процессов в смесях газов. Кинетический подход на уровне расчета функции распределения (ФР) необходим в задачах, где ФР не только сильно отклоняется от максвелловского распределения, но и, кроме того, является сильно анизотропной. К таким задачам относится кинетическое описание структуры ударных волн и нестационарных процессов их взаимодействия. Серьезной проблемой такого же рода является описание нестационарных процессов переноса в низкотемпературной плазме.

Одним из эффективных методов расчета функции распределения является моментный метод. В его основе лежит разложение ФР по набору базисных функций. Уравнение Больцмана при этом сводится к системе уравнений для коэффициентов разложения, а интеграл столкновений заменяется матрицей, элементы которой представляют собой коэффициенты разложения интеграла столкновений по базисным функциям.

Развитие моментного метода прежде всего связано с именем Барнетта [1,2]. В его работах фактически впервые выписана система нелинейных моментных уравнений. В качестве базисных функций была выбрана система ортогональных с максвелловским весом функций, представляющих собой произведения сферических гармоник и полиномов Сонина. В дальнейшем такой набор базисных функций получил название функций Барнетта. Были рассмотрены нелинейные матричные элементы (МЭ) интеграла столкновений в этом базисе. Как отмечал сам Барнетт, формулы для вычисления нелинейных МЭ получаются чрезвычайно громоздкими. Поэтому при конкретных расчетах Барнетт и его последователи (см., например, [3]) ограничились вычислениями для максвелловских молекул и модели твердых сфер при $l \leq 3$, где $l-$ порядок полинома Лежандра в разложении ФР.

В 1966 г. Кумар [4] проанализировал различные системы полиномов, которые использовались при разложении ФР в кинетической теории газов. Он показал, что наиболее экономичной является система функций Барнетта. Кумар продвинулся также в исследовании структуры нелинейного интеграла столкновений. Он предложил использовать при расчете нелинейных МЭ преобразование Талми, которое ранее успешно использовалось в квантовой теории.

Идеи Кумара нашли свое продолжение в работах ученых австралийской школы [5-8]. В этих работах моментный метод используется в основном для построения транспортных коэффициентов при движении заряженных частиц во внешних полях. Несмотря на то что в данном случае рассматривался линейный интеграл столкновений, до последнего времени не удавалось построить достаточное количество членов разложения ФР.

Проблема расчета МЭ с большими индексами остается актуальной. Так, относительно недавняя работа Шизгала с соавторами [9] посвящена расчету линейных изотропных МЭ. Разработанная авторами [9] процедура позволяет рассчитывать линейные изотропные МЭ с индексами $r \leq 50$, где $r-$ порядок полинома Сонина в разложении функции распределения.

В отличие от перечисленных выше работ, где строится несколько десятков моментов, нами был предложен метод последовательного определения МЭ, позволяющий находить десятки тысяч моментов ФР [10,11]. В качестве базисных функций также использовались функции Барнетта. При этом набор базисных функций определялся выбором температуры и средней скорости весового максвеллиана. Из фундаментального принципа инвариантности интеграла столкновений относительно выбора базиса были получены рекуррентные соотношения между МЭ, которые можно разделить на темпе- 
ратурные (следующие из инвариантности относительно выбора температуры) и скоростные (следующие из инвариантности относительно выбора величины средней скорости).

Скоростные соотношения являются чисто алгебраическими, в то время как температурные в общем случае включают производную по температуре. В случае степенных потенциалов взаимодействия все связи между МЭ (как скоростные, так и температурные) становятся чисто алгебраическими. Для таких сечений взаимодействия были развиты рекуррентные процедуры последовательного отыскания МЭ. При этом все нелинейные МЭ как изотропные (соответствующие изотропной по скоростям ФР), так и неизотропные, можно найти, если известны изотропные линейные МЭ.

Реализация такого подхода позволяет вычислять МЭ практически со сколь угодно большими индексами. Нами были табулированы матричные элементы для ряда степенных и квазистепенных законов взаимодействия частиц. В результате появилась возможность быстрого и точного расчета сильно неравновесных ФР в различных задачах, таких как нелинейная релаксация (ФР рассчитывалась вплоть до 10-20 тепловых скоростей [10]), и определение транспортных коэффициентов и ФР в сильных постоянных и переменных внешних полях. Отметим, что в последнем случае к значительному успеху привел переход к модифицированному моментному методу, в котором температура весового максвеллиана заряженных частиц отличается от температуры фонового газа и особым образом зависит от времени [12]. При таком подходе для расчета линейных МЭ в базисе с новой температурой необходимо некоторое подмножество нелинейных МЭ однотемпературного моментного метода.

Следует отметить, что двухтемпературный моментный метод широко используется в линейных задачах $[7,13,14]$, но предложенные там способы расчета МЭ не позволяют строить ФР, особенно в сильных полях и в высокоэнергетических областях.

Подчеркнем, что обсуждаемые задачи ранее в наших работах решались для модельных сечений рассеяния, соответствующих степенным и квазистепенным законам взаимодействия. Целью настоящей работа является обобщение процедуры построения МЭ на произвольные законы взаимодействия и произвольные массы взаимодействующих частиц.

Ниже будет показано, что все неизотропные МЭ могут быть найдены с помощью скоростных рекуррентных соотношений, если известны изотропные МЭ (как линейные, так и нелинейные).

В случае произвольных сечений взаимодействия температурные рекуррентные соотношения включают в себя производные по температуре, и для перехода к алгебраическим соотношениям удобно разложить изотропные МЭ по известным в кинетической теории $\Omega$-интегралам $[15,16]$. При этом рекуррентными соотношениями оказываются связаны не сами МЭ, а коэффициенты их разложения. Найденные таким образом изотропные МЭ являются стартовыми для скоростной рекуррентной процедуры, которая не зависит от потенциала взаимодействия. В результате рекуррентная процедура разбивается на две части: представление стартовых МЭ (линейных и нелинейных) в виде конечных сумм $\Omega$-интегралов и построение всех остальных матричных элементов с использованием алгебраических скоростных рекуррентных соотношений. Отметим, что во второй части рекуррентной процедуры рассмотрение ведется полностью на уровне матричных элементов, а не коэффициентов их разложения по $\Omega$-интегралам. Это позволяет существенно сократить количество индексов.

В этой работе будет построена рекуррентная процедура последовательного определения неизотропных МЭ, при этом стартовые изотропные МЭ будем считать известными. Их отысканию будет посвящена следующая работа, где они будут представлены в виде конечных сумм $\Omega$-интегралов.

\section{Основные соотношения}

Рассмотрим смесь двух сортов частиц $a$ и $b$ с массами $m_{a}$ и $m_{b}$. Нелинейные интегралы столкновений в этом случае имеют вид

$$
\begin{aligned}
\hat{I}\left(f_{a}, f_{b}\right)= & n_{a}(\mathbf{r}, t) n_{b}(\mathbf{r}, t) \int\left(f_{a}\left(\mathbf{v}_{1}\right) f_{b}\left(\mathbf{v}_{2}\right)\right. \\
& \left.-f_{a}(\mathbf{v}) f_{b}\left(\mathbf{v}^{\prime}\right)\right) g \sigma_{a b}(g, \theta) d \mathbf{v}^{\prime} d \mathbf{k} .
\end{aligned}
$$

Здесь $f_{a}, f_{b}$ - нормированные на единицу функции распределения по скоростям частиц сортов $a$ и $b, n_{a}$ и $n_{b}$ - плотности частиц. Скорости частиц до и после столкновения связаны соотношениями

$$
\begin{gathered}
\mathbf{v}_{1}=\mathbf{v}_{0}-\mu_{b} \mathbf{k} g, \quad \mathbf{v}_{2}=\mathbf{v}_{0}+\mu_{a} \mathbf{k} g, \quad \mathbf{v}_{0}=\left(\mu_{a} \mathbf{v}+\mu_{b} \mathbf{v}^{\prime}\right), \\
\mu_{a}=m_{a} /\left(m_{a}+m_{b}\right), \quad \mu_{b}=m_{b} /\left(m_{a}+m_{b}\right), \\
\mathbf{g}=\mathbf{v}_{1}-\mathbf{v}_{2}, \quad \mathbf{g}^{\prime}=\mathbf{v}-\mathbf{v}^{\prime}, \quad g=g^{\prime}
\end{gathered}
$$

где $\mathbf{k}-$ единичный вектор, направленный вдоль g. Угол рассеяния $\theta$ определяется соотношением $\cos \theta=\mathbf{k} \cdot \mathbf{g}^{\prime} / g$, а $\sigma_{a b}(g, \theta)$ - дифференциальное сечение рассеяния. Интеграл столкновений можно представить в форме

$$
\begin{aligned}
\hat{I}_{a, b}^{a}\left(f_{a}, f_{b}\right)= & n_{a} n_{b} \iint G_{a, b}^{a}\left(\mathbf{v}, \mathbf{v}_{1}, \mathbf{v}_{2}\right) \\
& \times f_{a}\left(\mathbf{v}_{1}\right) f_{b}\left(\mathbf{v}_{2}\right) d \mathbf{v}_{1} d \mathbf{v}_{2} .
\end{aligned}
$$

Здесь $G_{a, b}^{a}\left(\mathbf{v}, \mathbf{v}_{1}, \mathbf{v}_{2}\right)$ - ядро интеграла столкновений, зависящее от векторных скоростей. Это ядро содержит дельта-функцию, обеспечивающую выполнение закона сохранения энергии в столкновении [17].

Представим функцию распределения в виде разложения по сферическим гармоникам

$$
f_{a, b}(\mathbf{v})=\sum_{l=0}^{\infty} \sum_{m=0}^{l} \sum_{i=0}^{1}\left(f_{a, b}\right)_{l, m}^{i}(v) Y_{l, m}^{i}(\Theta, \varphi),
$$




$$
\begin{gathered}
Y_{l m}^{0}(\Theta, \varphi)=P_{l}^{m}(\cos \Theta) \cos m \varphi, \\
Y_{l m}^{1}(\Theta, \varphi)=P_{l}^{m}(\cos \Theta) \sin m \varphi, \\
0 \leq m \leq l .
\end{gathered}
$$

Здесь $P_{l}^{m}(\cos \Theta)$ - присоединенные полиномы Лежандpa, $\Theta, \varphi-$ угловые координаты вектора скорости v. При использовании разложения (4) интеграл столкновений принимает вид

$$
\begin{aligned}
& \hat{I}\left(f_{a}, f_{b}\right)=n_{a}(\mathbf{r}, t) n_{b}(\mathbf{r}, t) \\
& \times \int_{0}^{\infty} \int_{0}^{\infty}\left(\sum_{l_{1}, l_{2}, m_{1}, m_{2}, i_{1}, i_{2}}\left(G_{a, b}^{a}\right)_{l_{1}, m_{1}, i_{1}, l_{2}, m_{2}, i_{2}}^{l, m, i}\left(v, v_{1}, v_{2}\right)\right. \\
& \left.\times\left(f_{a}\right)_{l_{1}, m_{1}}^{i_{1}}\left(v_{1}\right)\left(f_{b}\right)_{l_{2}, m_{2}}^{i_{2}}\left(v_{2}\right)\right) v_{1}^{2} v_{2}^{2} d v_{1} d v_{2} .
\end{aligned}
$$

Как видно из (5), пятикратный интеграл столкновений заменяется суммой более простых, двукратных, интегральных операторов. Ядра этих операторов

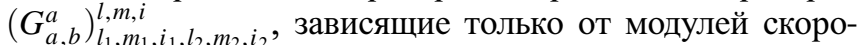
стей $v, v_{1}, v_{2}$, являются проекциями ядра, зависящего от векторных скоростей, на сферические гармоники.

При дальнейшем разложении $\left(f_{a, b}\right)_{l, m}^{i}(v)$ по базисным функциям, зависящим от модуля скорости, интеграл столкновений заменяется числовой матрицей. В кинетической теории в качестве таких базисных функций часто используют полиномы Сонина (Лагерра) $S_{l+1 / 2}^{r}\left(x^{2}\right)$. При этом разложение ФР приобретает вид

$$
\begin{gathered}
f_{a}\left(\mathbf{c}_{a}, \mathbf{r}, t\right)=M_{a} \sum C_{r l m}^{i, a}(\mathbf{r}, t) H_{r l m}^{i}\left(\mathbf{c}_{a}\right), \\
\mathbf{c}_{a}=\sqrt{\frac{m_{a}}{2 k T}}(\mathbf{v}-\mathbf{u}), \\
M_{a}=\left(\frac{m_{a}}{2 k T \pi}\right)^{3 / 2} e^{-c_{a}^{2},} \\
H_{j}\left(\mathbf{c}_{a}\right)=Y_{l m}^{i}(\Theta, \varphi) c_{a}^{l} S_{l+1 / 2}^{r}\left(c_{a}^{2}\right), \quad i=0,1 .
\end{gathered}
$$

Здесь индекс $j$ состоит из четырех индексов $(r, l, m, i)$, $M_{a}$ - весовой максвеллиан с температурой $T$ и средней скоростью и. Аналогично записывается разложение функции распределения $b$-го компонента $f_{b}$.

Уравнение Больцмана в этом случае заменяется бесконечной системой моментных уравнений. Для коэффициентов разложения ФР $a$-го компонента смеси $C_{j}^{a}$ имеем

$$
\frac{D_{M}\left(C_{j}^{a}\right)}{D t}=\sum_{j_{1}, j_{2}}\left(K_{a, b}^{a}\right)_{j_{1}, j_{2}}^{j} C_{j_{1}}^{a} C_{j_{2}}^{b}+\sum_{j_{1}, j_{2}}\left(K_{a, a}^{a}\right)_{j_{1}, j_{2}}^{j} C_{j_{1}}^{a} C_{j_{2}}^{a} .
$$

Явный вид дифференциального оператора моментной системы $D_{M}\left(C_{j}^{a}\right) / D t$ можно найти в [18]. Нелинейные матричные элементы интеграла столкновений $\left(K_{a, b}^{a}\right)_{j_{1}, j_{2}}^{j}$ определяются следующим образом:

$$
\begin{gathered}
\left(K_{a, b}^{a}\right)_{j_{1}, j_{2}}^{j}=\int H_{j}\left(\mathbf{c}_{a}\right) \hat{I}\left(M_{a} H_{j_{1}}\left(\mathbf{c}_{a}\right), M_{b} H_{j_{2}}\left(\mathbf{c}_{b}\right)\right) d^{3} v / g_{j} \\
g_{j}=\int \pi^{-3 / 2} e^{-c^{2}} H_{j}^{2}(\mathbf{c}) d^{3} c
\end{gathered}
$$

Они связаны с ядрами соотношением

$$
\begin{aligned}
& \left(K_{a, b}^{a}\right)_{j_{1}, j_{2}}^{j}=\iiint\left(G_{a, b}^{a}\right)_{l_{1}, m_{1}, i_{1}, l_{2}, m_{2}, i_{2}}^{l, m, i}\left(v, v_{1}, v_{2}\right) \\
& \times S_{l+1 / 2}^{r}\left(c^{2}\right) S_{l_{1}+1 / 2}^{r}\left(c_{1}^{2}\right) S_{l_{2}+1 / 2}^{r}\left(c_{2}^{2}\right) c^{2} c_{1}^{2} c_{2}^{2} d c d c_{1} d c_{2} / \sigma_{r l},
\end{aligned}
$$

где $M(c)=\pi^{-3 / 2} e^{-c^{2}}$

Отметим тот факт ([11]), что для любых неориентированных в пространстве частиц (для сечений взаимодействия, зависящих только от двух переменных - модуля относительной скорости и угла рассеяния) произвольные МЭ пропорциональны соответствующим осесимметричным МЭ (т. е. МЭ с индексами $m=m_{1}=m_{2}=0$ ):

$$
\left(K_{a, b}^{a}\right)_{r_{1}, l_{1}, m_{1}, i_{1}, r_{2}, l_{2}, m_{2}, i_{2}}^{r, l, m, i}=\check{Z}_{l_{1} m_{1} i_{1}, l_{2} m_{2} i_{2}}^{l m i}\left(K_{a, b}^{a}\right)_{r_{1}, l_{1}, r_{2}, l_{2}}^{r, l} .
$$

Здесь и далее опускаем индексы $m, i$ в обозначениях осесимметричных МЭ. Существенно, что числа $\check{Z}_{l_{1} m_{1} i_{1}, l_{2} m_{2} i_{2}}^{l m}$ могут отличаться от нуля, только если

$$
\left|l_{l}-l_{2}\right| \leq l \leq l_{1}+l_{2}, \quad(-1)^{l+l_{1}+l_{2}}=1 .
$$

Кроме того, должны выполняться условия $m=\mid m_{1} \pm$ $\pm m_{2} \mid,(-1)^{i+i_{1}+i_{2}}=1, m \leq l, m_{1} \leq l_{1}, m_{2} \leq l_{2}$. Эти условия вместе с (12) составляют обобщенную теорему Гекке (ОТГ) [11,19], которая распространяет теорему Гекке $[20,21]$ на нелинейный случай. Для линейных МЭ, когда $j_{1}$ или $j_{2}$ равны нулю, $\check{Z}=1$ и соответственно либо $\left(l_{2}, m_{2}, i_{2}\right)=(l, m, i)$, либо $\left(l_{1}, m_{1}, i_{1}\right)=(l, m, i)$.

При разложении изотропного по скоростям интеграла столкновений отличны от нуля только матричные элементы вида $K_{r_{1}, 0, r_{2}, 0}^{r, 0}$, которые в дальнейшем будем называть изотропными. Матричные элементы, у которых хотя бы один из индексов $l, l_{1}, l_{2}$ отличен от нуля, будем называть неизотропными.

Линейные МЭ соответствуют линейному интегралу столкновений и могут быть первого $\left(\left(K_{a, b}^{a}\right)_{j, 0}^{i}\right)$ и второго $\left(\left(K_{a, b}^{a}\right)_{0, k}^{i}\right)$ типов. Обозначим их $\left(\Lambda_{a, b}^{a}\right)$. В осесимметричном случае имеем

$$
\left(\Lambda_{a, b}^{a}\right)_{r, r_{1}, l}^{(1)}=\left(K_{a, b}^{a}\right)_{r_{1}, l, 0,0}^{r, l} ; \quad\left(\Lambda_{a, b}^{a}\right)_{r, r_{2}, l}^{(2)}=\left(K_{a, b}^{a}\right)_{0,0, r_{2}, l}^{r, l} .
$$

Используя (10) и (11), легко показать, что ядра интеграла столкновений, полученные при проектировании $G_{a, b}^{a}\left(\mathbf{v}, \mathbf{v}_{1}, \mathbf{v}_{2}\right)$ на сферические гармоники, представляют собой линейные комбинации осесимметричных ядер $\left(G_{a, b}^{a}\right)_{l_{1}, l_{2}}^{l}\left(v, v_{1}, v_{2}\right)$ :

$$
\begin{aligned}
& \left(G_{a, b}^{a}\right)_{l_{1}, m_{1}, i_{1}, l_{2}, m_{2}, i_{2}}^{l, m, v_{1}}\left(v, v_{1}, v_{2}\right) \\
& =\check{Z}_{m_{1}, i_{1}, m_{2}, i_{2}}^{m, i}\left(l, l_{1}, l_{2}\right)\left(G_{a, b}^{a}\right)_{l_{1}, l_{2}}^{l}\left(v, v_{1}, v_{2}\right) .
\end{aligned}
$$

Ядра $\left(G_{a, b}^{a}\right)_{l_{1}, l_{2}}^{l}$ определяются как коэффициенты разложения ядра $G_{a, b}^{a}\left(\mathbf{v}, \mathbf{v}_{1}, \mathbf{v}_{2}\right)$ по полиномам Лежандра

$$
\begin{aligned}
& \left(G_{a, b}^{a}\right)_{l_{1}, l_{2}}^{l}\left(v, v_{1}, v_{2}\right)=4 \pi \int_{-1}^{1} \int_{-1}^{1} \int_{-1}^{1} \frac{P_{l}(\cos \Theta)}{\left\|P_{l}\right\|^{2}} \\
& \times G_{a, b}^{a}\left(\mathbf{v}, \mathbf{v}_{1}, \mathbf{v}_{2}\right) P_{l_{1}}\left(\cos \Theta_{1}\right) \\
& \times P_{l_{2}}\left(\cos \Theta_{2}\right) d \cos \Theta d \cos \Theta_{1} d \cos \Theta_{2} .
\end{aligned}
$$


Из написанного выше следует, что ядра (15) могут быть представлены в виде

$$
\begin{aligned}
& \left(G_{a, b}^{a}\right)_{l_{1}, l_{2}}^{l}\left(v, v_{1}, v_{2}\right)=M(c) \sum_{r, r_{1}, r_{2}} c^{l} S_{l+1 / 2}^{r}\left(c^{2}\right) \\
& \times\left(K_{a, b}^{a}\right)_{r_{1}, l_{1}, r_{2}, l_{2}}^{r, l} \frac{c_{1}^{l_{1}} S_{l_{1}+1 / 2}^{r_{l}}\left(c_{1}^{2}\right)}{\sigma_{r_{1} l_{1}}} \frac{c_{2}^{l_{2}} S_{l_{2}+1 / 2}^{r_{2}}\left(c_{2}^{2}\right)}{\sigma_{r_{2} l_{2}}},
\end{aligned}
$$

где $\sigma_{r l}-$ нормировочные множители полиномов Сонина.

В $[11,22]$ из инвариантности интеграла столкновений относительно выбора температуры и средней скорости весового максвеллиана были получены две группы рекуррентных соотношений: температурные

$$
\begin{aligned}
& \left(T \frac{d}{d T}+r-r_{1}-r_{2}-\frac{l_{1}+l_{2}-l}{2}\right)\left(K_{a, b}^{a}\right)_{r_{1}, l_{1}, r_{2}, l_{2}}^{r, l} \\
& =r\left(K_{a, b}^{a}\right)_{r_{1}, l_{1}, r_{2}, l_{2}}^{r-1}-\left(r_{1}+1\right)\left(K_{a, b}^{a}\right)_{r_{1}+1, l_{1}, r_{2}, l_{2}}^{r, l} \\
& -\left(r_{2}+1\right)\left(K_{a, b}^{a}\right)_{r_{1}, l_{1}, r_{2}+1, l_{2}}^{r, l}
\end{aligned}
$$

и скоростные

$$
\begin{aligned}
& \beta(l-1)\left(K_{a, b}^{a}\right)_{r_{1}, l_{1}, r_{2}, l_{2}}^{r, l}+\gamma(r-1, l+1)\left(K_{a, b}^{a}\right)_{r_{1}, l_{1}, r_{2}, l_{2}}^{r-1, l+1} \\
& -\beta\left(l_{1}\right)\left(K_{a, b}^{a}\right)_{r_{1}, l_{1}+1, r_{2}, l_{2}}^{r, l}-\gamma\left(r_{1}, l_{1}\right)\left(K_{a, b}^{a}\right)_{r_{1}+1, l_{1}-1, r_{2}, l_{2}}^{r, l} \\
& -\sqrt{\frac{m_{b}}{m_{a}}}\left(\beta\left(l_{2}\right)\left(K_{a, b}^{a}\right)_{r_{1}, l_{1}, r_{2}, l_{2}+1}^{r, l}\right. \\
& \left.+\gamma\left(r_{2}, l_{2}\right)\left(K_{a, b}^{a}\right)_{r_{1}, l_{1}, r_{2}+1, l_{2}-1}^{r, l}\right)=0 \\
& \beta(l)=-\frac{l+1}{2 l+1}, \quad \gamma(r, l)=\frac{(r+1) l}{2 l+1} .
\end{aligned}
$$

На основе (17), (18) для степенных законов взаимодействия в $[11,22]$ была разработана рекуррентная процедура с использованием простого аналитического выражения для линейных изотропных МЭ. Для произвольных потенциалов взаимодействия предлагается видоизмененная рекуррентная процедура. При этом расчет разбивается на два этапа. На первом этапе температурные соотношения используются для построения линейных и нелинейных изотропных МЭ с помощью разложения по $\Omega$-интегралам. При этом рекуррентные соотношения для МЭ сводятся к алгебраическим соотношениям для коэффициентов разложения. Наиболее экономичным для расчета оказывается использование в качестве стартовых коэффициентов разложения линейных изотропных МЭ второго типа $\left(\Lambda_{a, b}^{a}\right)_{r, r_{2}, l}^{(2)}$. На втором этапе найденные изотропные МЭ используются в качестве стартовых для построения неизотропных МЭ с ненулевыми индексами $l, l_{1}, l_{2}$. Таким образом, конкретный вид сечения взаимодействия используется только на этапе построения стартовых линейных изотропных МЭ второго типа. В этой работе будет изложена вторая, универсальная часть алгоритма. Построению стартовых изотропных МЭ будет посвящена наша следующая работа.

\section{Построение неизотропных матричных элементов}

Покажем, что скоростные соотношения (18) позволяют последовательно выражать МЭ с произвольными индексами непосредственно через изотропные МЭ без обращения к $\Omega$-интегралам на каждом шаге второго этапа рекуррентной процедуры. Для краткости, в обозначениях МЭ будем опускать индексы $a, b$, указывающие сорт взаимодействующих частиц.

Введем, как и в [11], параметры $R, p, q, \lambda, \tau, \nu$ :

$$
\begin{aligned}
& R=r_{1}-r+\left(l_{1}+l_{2}-l\right) / 2, \quad p=r_{1}, \quad q=r_{2}, \\
& \lambda=\left(l_{1}+l_{2}+l\right) / 2, \quad \tau=\left(l-l_{1}+l_{2}\right) / 2, \quad v=l_{2} .
\end{aligned}
$$

Легко выразить исходные индексы через эти параметры:

$$
\begin{gathered}
r=p-R-\tau+v, \quad r_{1}=p, \quad r_{2}=q, \\
l=\lambda-v+\tau, \quad l_{1}=\lambda-\tau, \quad l_{2}=v .
\end{gathered}
$$

Из неотрицательности $l, l_{1}, l_{2}$ следует, что $\lambda \geq 0$, $v \geq 0$, а из четности суммы $l+l_{1}+l_{2}$ (см. (12)) следует, что $\lambda, v$ - целые. Можно показать, что остальные условия ОТГ (12) эквивалентны неравенствам

$$
0 \leq \tau \leq v \leq \lambda
$$

Заменим в соотношении (18) $l_{2}$ на $l_{2}-1$ и перегруппируем слагаемые таким образом, чтобы выразить $K_{r_{1}, l_{1}, r_{2}, l_{2}}^{r, l}$ через остальные МЭ, после чего перепишем (18), используя введенные параметры:

$$
\begin{aligned}
& \sqrt{\frac{m_{b}}{m_{a}}} \beta(v-1) K_{p, \lambda-\tau, q, v}^{p-R-\tau+v, \lambda-v+\tau} \\
& =\gamma(p-R-\tau+v-1, \lambda-v+\tau+1) K_{p, \lambda-\tau, q, v-1}^{p-R-\tau+v-1, \lambda-v+\tau+1} \\
& -\beta(\lambda-\tau) K_{p, \lambda-\tau+1, q, v-1}^{p-R-\tau+\nu, \lambda-v+\tau}+\{\beta(\lambda-v+\tau-1) \\
& \times K_{p, \lambda-\tau, q, v-1}^{p-R-\tau+v, \lambda-v+\tau-1}-\gamma(p, \lambda-\tau) K_{p+1, \lambda-\tau-1, q, v-1}^{p-R-\tau+v, \lambda-v+\tau} \\
& \left.-\sqrt{\frac{m_{b}}{m_{a}}} \gamma(q, v-1) K_{p, \lambda-\tau, q+1, \nu-2}^{p-R-\tau+v, \lambda-v+\tau}\right\} .
\end{aligned}
$$

Отметим, что в фигурных скобках стоят МЭ, у которых сумма индексов, соответствующих разложению по полиномам Лежандра, равна $2 \lambda-2$, т.е. на 2 меньше, чем сумма тех же индексов МЭ вне фигурных скобок, которая равна $\lambda$. Ниже матричные элементы и ядра с суммой индексов $l+l_{1}+l_{2}=\lambda$ будем называть слоем $\lambda$. Обозначим выражение в фигурных скобках $Q_{p, \lambda, q}^{R}(\tau, v)$. Будем считать, что оно найдено на предыдущем шаге рекуррентной процедуры. На первом шаге в фигурных скобках стоят изотропные МЭ, которые являются стартовыми. Фиксируем параметры $\lambda, p, q$ и $R \leq p$. Положим 
$\tau=0$ и запишем соотношение (22) для $v \geq 1$ (при $v=0$ левая и правая части равенства обращаются в нуль):

$$
\begin{aligned}
& \sqrt{\frac{m_{b}}{m_{a}}} \beta(v-1) K_{p, \lambda, q, v}^{p-R+v, \lambda-v} \\
& =\gamma(p-R+v-1, \lambda-v+1) K_{p, \lambda, q, v-1}^{p-R+v-1, \lambda-v+1} \\
& -\beta(\lambda) K_{p, \lambda+1, q, v-1}^{p-R+v, \lambda-v}+Q_{p, \lambda, q}^{R}(0, v) .
\end{aligned}
$$

Второй член справа в этом равенстве равен нулю, поскольку в силу (12) МЭ с индексами, не удовлетворяющими неравенству $\left|l_{1}-l_{2}\right| \leq l$, обращаются в нуль. Используя метод математической индукции, легко показать, что при $1 \leq v \leq \lambda$ равенство (23) можно переписать в виде

$$
K_{p, \lambda, q, v}^{p-R+v, \lambda-v}=L_{p, \lambda, q}^{R}(0, v) K_{p, \lambda, q, 0}^{p-R, \lambda}+M_{p, \lambda, q}^{R}(0, v),
$$

где

$$
\begin{gathered}
L_{p, \lambda, q}^{R}(0, v)=\frac{\gamma(p-R+v-1, \lambda-v+1)}{\beta(v-1) \sqrt{m_{b} / m_{a}}} L_{p, \lambda, q}^{R}(0, v-1), \\
M_{p, \lambda, q}^{R}(0, v)=\frac{\gamma(p-R+v-1, \lambda-v+1)}{\beta(v-1) \sqrt{m_{b} / m_{a}}} \\
\quad \times M_{p, \lambda, q}^{R}(0, v-1)+\frac{Q_{p, \lambda, q}^{R}(0, v)}{\beta(v-1) \sqrt{m_{b} / m_{a}}}
\end{gathered}
$$

То есть все МЭ вида $K_{p, \lambda, q, v}^{p-R+v, \lambda-v}$ выражаются через $K_{p, \lambda, q, 0}^{p-R, \lambda}$ и известные функции $Q_{p, \lambda, q}^{R}(0, v)$. Положим теперь в основном соотношении $(22) \tau=1$ :

$$
\begin{aligned}
& \beta(v-1) \sqrt{m_{b} / m_{a}} K_{p, \lambda-1, q, v}^{p-R-1+v, \lambda-v+1} \\
& =\gamma(p-R+v-2, \lambda-v+2) K_{p, \lambda-1, q, v-1}^{p-R+v-2, \lambda-v+2} \\
& -\beta(\lambda-1) K_{p, \lambda, q, v-1}^{p-R-1+v, \lambda-v+1}+Q_{p, \lambda, q}^{R}(1, v) .
\end{aligned}
$$

При $v=1$ имеем

$$
\begin{aligned}
& \beta(0) \sqrt{m_{b} / m_{a}} K_{p, \lambda-1, q, 1}^{p-R, \lambda}=\gamma(p-R-1, \lambda+1) K_{p, \lambda-1, q, 0}^{p-R-1, \lambda+1} \\
& -\beta(\lambda-1) K_{p, \lambda, q, 0}^{p-R, \lambda}+Q_{p, \lambda, q}^{R}(1,1) .
\end{aligned}
$$

Первый член справа обращается в нуль вследствие (12), поскольку для индексов МЭ нарушается условие $l \leq l_{1}+l_{2}$. Таким образом, $K_{p, \lambda-1, q, 1}^{p-R, \lambda}$ представляется в виде

$$
K_{p, \lambda-1, q, 1}^{p-R, \lambda}=L_{p, \lambda, q}^{R}(1,1) K_{p, \lambda, q, 0}^{p-R, \lambda}+M_{p, \lambda, q}^{R}(1,1),
$$

где

$$
\begin{aligned}
& L_{p, \lambda, q}^{R}(1,1)=-\frac{\beta(\lambda-1)}{\beta(0) \sqrt{m_{b} / m_{a}}}, \\
& M_{p, \lambda, q}^{R}(1,1)=\frac{Q_{p, \lambda, q}^{R}(1,1)}{\beta(0) \sqrt{m_{b} / m_{a}}} .
\end{aligned}
$$

Последовательно увеличивая $v$ в диапазоне $1<v \leq \lambda$, методом математической индукции легко показать, что все МЭ вида $K_{p, \lambda-1, q, v}^{p-R+v-1, \lambda-(v-1)}$ также можно выразить через $K_{p, \lambda, q, 0}^{p-R, \lambda}$ и известные функции $Q_{p, \lambda, q}^{R}(1, v)$ :

$$
K_{p, \lambda-1, q, v}^{p-R+v-1, \lambda-(v-1)}=L_{p, \lambda, q}^{R}(1, v) K_{p, \lambda, q, 0}^{p-R, \lambda}+M_{p, \lambda, q}^{R}(1, v),
$$

где

$$
\begin{aligned}
& L_{p, \lambda, q}^{R}(1, v)=\frac{\gamma(p-R+v-2, \lambda-v+2)}{\beta(v-1) \sqrt{m_{b} / m_{a}}} L_{p, \lambda, q}^{R}(1, v-1) \\
& -\frac{\beta(\lambda-1)}{\beta(v-1) \sqrt{m_{b} / m_{a}}} L_{p, \lambda, q}^{R}(0, v-1), \\
& M_{p, \lambda, q}^{R}(1, v)=\frac{\gamma(p-R+v-2, \lambda-v+2)}{\beta(v-1) \sqrt{m_{b} / m_{a}}} M_{p, \lambda, q}^{R}(1, v-1) \\
& -\frac{\beta(\lambda-1)}{\beta(v-1) \sqrt{m_{b} / m_{a}}} M_{p, \lambda, q}^{R}(0, v-1)+\frac{Q_{p, \lambda, q}^{R}(1, v)}{\beta(v-1) \sqrt{m_{b} / m_{a}}} .
\end{aligned}
$$

При $v=\lambda+1$ МЭ $K_{p, \lambda-1, q, \lambda+1}^{p-R+\lambda, 0}$, стоящий в левой части рекуррентного соотношения, обращается в нуль, что дает уравнение для определения $K_{p, \lambda, q, 0}^{p-R, \lambda}$ :

$$
L_{p, \lambda, q}^{R}(1, \lambda+1) K_{p, \lambda, q, 0}^{p-R, \lambda}+M_{p, \lambda, q}^{R}(1, \lambda+1)=0 .
$$

Отсюда

$$
K_{p, \lambda, q, 0}^{p-R, \lambda}=-\frac{M_{p, \lambda, q}^{R}(1, \lambda+1)}{L_{p, \lambda, q}^{R}(1, \lambda+1)} .
$$

Теперь из соотношений (24), (29), (31) находятся все МЭ, соответствующие $\tau=0,1$.

Чтобы найти МЭ, отвечающие другим значениям $\tau$, рассмотрим основное соотношение при $\tau>1$. При $v=\tau$ соотношение (22) перейдет в

$$
\begin{aligned}
& \beta(\tau-1) \sqrt{\frac{m_{b}}{m_{a}}} K_{p, \lambda-\tau, q, \tau}^{p-R, \lambda}=\gamma(p-R-1, \lambda+1) K_{p, \lambda-\tau, q, \tau-1}^{p-R-1, \lambda+1} \\
& -\beta(\lambda-\tau) K_{p, \lambda-\tau+1, q, \tau-1}^{p-R, \lambda}+Q_{p, \lambda, q}^{R}(\tau, \tau) .
\end{aligned}
$$

Первый член справа обращается в нуль в силу (12) (сумма нижних индексов МЭ меньше верхнего), и

$$
K_{p, \lambda-\tau, q, \tau}^{p-R, \lambda}=L_{p, \lambda, q}^{R}(\tau, \tau) K_{p, \lambda-\tau+1, q, \tau-1}^{p-R, \lambda}+M_{p, \lambda, q}^{R}(\tau, \tau),
$$

где

$$
\begin{gathered}
L_{p, \lambda, q}^{R}(\tau, \tau)=-\frac{\beta(\lambda-\tau)}{\beta(\tau-1) \sqrt{m_{b} / m_{a}}}, \\
M_{p, \lambda, q}^{R}(\tau, \tau)=\frac{Q_{p, \lambda, q}^{R}(\tau, \tau)}{\beta(\tau-1) \sqrt{m_{b} / m_{a}}} .
\end{gathered}
$$

Меняя $\tau$ от 2 до $\lambda$, последовательно найдем МЭ вида $K_{p, \lambda-\tau, q, \tau}^{p-R, \lambda}$, поскольку $K_{p, \lambda-1, q, 1}^{p-R, \lambda}$ известен. Используем теперь основное соотношение (22) при $v>\tau$. Оно имеет 
вид

$$
\begin{aligned}
& K_{p, \lambda-\tau, q, v}^{p-R-\tau+v, \lambda-v+\tau}=\frac{\gamma(p-R-\tau+v-1, \lambda-v+\tau+1)}{\beta(v-1) \sqrt{m_{b} / m_{a}}} \\
& \times K_{p, \lambda-\tau, q, v-1}^{p-R-\tau+v-1, \lambda-v+\tau+1}-\frac{\beta(\lambda-\tau)}{\beta(v-1) \sqrt{m_{b} / m_{a}}} \\
& \times K_{p, \lambda-\tau+1, q, v-1}^{p-R-\tau+v, \lambda-v+\tau}+\frac{Q_{p, \lambda, q}^{R}(\tau, v)}{\beta(v-1) \sqrt{m_{b} / m_{a}}} .
\end{aligned}
$$

При $\tau$ в диапазоне от 2 до $\lambda$, увеличивая $v$ от $\tau+1$ до $\lambda$, найдем МЭ вида $K_{p, \lambda-\tau, q, v}^{p-R-\tau+v-\nu+\tau}$, поскольку МЭ, входящий в первый член в правой части (36), получен на предыдущем шаге по $v$, а второй - на предыдущем шаге по $\tau$.

Чтобы найти МЭ со всеми комбинациями индексов, нужно выполнять описанную выше процедуру при всех возможных $p$ и $q$. Отметим, что в функции $Q_{p, \lambda, q}^{R}(\tau, v)$ в (22) входят МЭ с индексами $p+1, q+1$. Это означает, что на каждом следующем шаге по $\lambda$ диапазон изменения параметров $p$ и $q$ уменьшается на единицу. Если известны стартовые изотропные МЭ с индексами $0 \leq r_{1} \leq N, 0 \leq r_{2} \leq N$, то при фиксированном значении $\lambda$ можно найти МЭ с индексами $0 \leq r_{1} \leq N-\lambda$, $0 \leq r_{2} \leq N-\lambda$.

Выше предполагалось, что $R \leq p$, и МЭ $K_{p, \lambda, q, 0}^{p-R, \lambda}$ в соотношениях (24), (29), (31) отличен от нуля. Это соответствует условию $v-\tau \leq r$. Чтобы получить МЭ с индексами $0 \leq r<v-\tau$, нужно рассматривать $R$ в диапазоне $p<R \leq p+(v-\tau)$. По аналогии с описанной выше процедурой будем при фиксированных $p, q$ и $R>p$ последовательно увеличивать $\tau$ от нуля до $\lambda$, а $v-$ от $R-p+\tau$ до $\lambda$.

Рассмотрим соотношение (23), в которое переходит основное соотношение (22) при $\tau=0$. При $v=R-p$ первый член в правой части (23) равен нулю, и

$$
K_{p, \lambda, q, R-p}^{0, \lambda-R+p}=\frac{Q_{p, \lambda, q}^{R}(0, R-p)}{\beta(R-p-1) \sqrt{m_{b} / m_{a}}} .
$$

Для $R-p+1 \leq v \leq \lambda$ МЭ вида $K_{p, \lambda, q, v}^{p-R+v, \lambda-v}$ последовательно вычисляются с помощью соотношения (24). Заметим, что при $v=\lambda+1$ правая часть (23) должна обращаться в нуль.

Рассмотрим теперь основное соотношение при $1 \leq$ $\leq \tau \leq \lambda$. В том случае, когда $v=R-p+\tau$, соотношение (22) переходит в (36), причем первый член справа равен нулю, а второй член известен. Поэтому

$$
\begin{aligned}
& K_{p, \lambda-\tau, q, R-p+\tau}^{0, \lambda-R+p}=-\frac{\beta(\lambda-\tau)}{\beta(R-p+\tau-1) \sqrt{m_{b} / m_{a}}} \\
& \times K_{p, \lambda-\tau+1, q, R-p+\tau-1}^{0, \lambda-R+p}+\frac{Q_{p, \lambda, q}^{R}(\tau, R-p+\tau)}{\beta(R-p+\tau-1) \sqrt{m_{b} / m_{a}}} .
\end{aligned}
$$

При $R-p+\tau \leq v \leq \lambda$ МЭ последовательно находятся из соотношения (36). При $v=\lambda+1$ МЭ должны быть равны нулю.
Таким образом, все МЭ с индексами, отвечающими слою $\lambda$, могут быть найдены из рекуррентной процедуры, базирующейся на скоростных соотношениях. Отметим, что при такой организации рекуррентной процедуры на этапе построения неизотропных МЭ температурные соотношения (17) не используются. В $[11,18]$ была описана рекуррентная процедура расчета МЭ в случае степенных законов взаимодействия. В отличие от алгоритма, описанного выше, она включала использование температурных соотношений (17) при построении неизотропных МЭ. Это связано с тем, что в случае степенных потенциалов соотношения (17) превращаются в алгебраические. На примере модели твердых шаров было проведено сравнение МЭ, рассчитанных с помощью этих двух алгоритмов (предложенного выше и описанного в $[11,18])$, и проверено совпадение результатов.

\section{Температурные рекуррентные соотношения}

Подчеркнем еще раз, что в описываемой здесь процедуре температурные рекуррентные соотношения прямо не использовались. Таким образом, они являются дополнительными связями между построенными МЭ. Докажем, что (17) удовлетворяются тождественно и обе процедуры (описанная в $[11,18]$ для степенных потенциалов и предлагаемая в данной работе) эквиваленты. С этой целью покажем, что если стартовые изотропные МЭ удовлетворяют температурным соотношениям (17), то и все МЭ удовлетворяют этим соотношениям.

Заметим, что найденные на слое $\lambda$ матричные элементы позволяют определить ядра интеграла столкновений с помощью (16). При этом свойство (17) эквивалентно условию

$$
\left(2 T \frac{\partial}{\partial T}-c \frac{\partial}{\partial c}-c_{1} \frac{\partial}{\partial c_{1}}-c_{2} \frac{\partial}{\partial c_{2}}\right) G_{l_{1}, l_{2}}^{l}=0 .
$$

Чтобы показать это, достаточно подставить (16) в (39) и воспользоваться свойством полиномов Лагерра $L_{r}^{\alpha}(x)[23]$

$$
\begin{aligned}
x \frac{d L_{r}^{\alpha}(x)}{d x}= & n L_{r}^{\alpha}(x)-(n+\alpha) L_{r-1}^{\alpha}(x)=(n+1) L_{r+1}^{\alpha}(x) \\
& -(n+\alpha+1-x) L_{r}^{\alpha}(x) .
\end{aligned}
$$

Полиномы Лагерра $L_{r}^{\alpha}(x)$ совпадают с полиномами Сонина $S_{l+1 / 2}^{r}(x)$ при $\alpha=l+1 / 2$, где $l$ - целое. Поскольку изотропные МЭ удовлетворяют (17), ядро $G_{0,0}^{0}$ удовлетворяет соотношению (39). Покажем, что если (39) справедливо для ядер на слое $\lambda-1$, то оно справедливо и для ядер на слое $\lambda$ и, следовательно, соответствующие матричные элементы удовлетворяют температурным соотношениям.

Как показано в [24], для ядер справедливы соотношения, связывающие ядра на слое $\lambda$ с ядрами на слое $\lambda-1$. 
В частности, для ядра $G_{\lambda, 0}^{\lambda}$ получено выражение [25]

$$
\begin{aligned}
& G_{\lambda, 0}^{\lambda}=\frac{(-1)^{\lambda+1}}{(\lambda+1)}\left(\hat{B}_{\lambda-1}^{(2)}(c)\right)^{-1} \ldots\left(\hat{B}_{1}^{(2)}(c)\right)^{-1}\left(\hat{B}_{0}^{(2)}(c)\right)^{-1} \\
& \times\left(\hat{B}_{\lambda-1}^{(3)}\left(c_{1}\right)\right)^{-1}\left(\hat{B}_{0}^{(3)}\left(c_{2}\right) \ldots \hat{B}_{\lambda-2}^{(3)}\left(c_{2}\right) \hat{B}_{\lambda-1}^{(3)}\left(c_{2}\right)\right) \\
& \times\left(\hat{B}_{0}^{(2)}(c) \tilde{M}_{\lambda}(1, \lambda)+\hat{B}_{\lambda-1}^{(3)}\left(c_{1}\right) \tilde{M}_{\lambda}(0, \lambda)\right.
\end{aligned}
$$$$
\left.+\hat{B}_{\lambda}^{(4)}\left(c_{2}\right) G_{\lambda-1, \lambda-1}^{0}\right)
$$

где операторы $\hat{B}_{j}^{(i)}(c),\left(\hat{B}_{j}^{(i)}(c)\right)^{-1}$ определяются выражениями

$$
\begin{aligned}
& \hat{B}_{j}^{(i)}(c) f(c)=a(i, j)\left(\frac{\partial}{\partial c}+\frac{b(i, j)}{c}\right) f(c), \\
& \left(\hat{B}_{j}^{(i)}(c)\right)^{-1} f(c)=\frac{a(i, j)}{c^{b(i, j)}} \int_{0}^{c} t^{b(i, j)} f(t) d t,
\end{aligned}
$$

$a(1, j)=j /(2 j-1)$, $a(3, j)=(j+1) /(2 j+1)$,

$$
a(2, j)=(j+1) /(2 j+3),
$$
$a(4, j)=j /(2 j+1)$, $b(1, j)=b(4, j)=j-1, \quad b(2, j)=b(3, j)=j+2$. Функции $\tilde{M}_{\lambda}(0, \lambda), \tilde{M}_{\lambda}(1, \lambda)$ находятся из рекуррентных соотношений

$$
\begin{gathered}
\tilde{M}_{\lambda}(0,1)=-\left(\hat{B}_{0}^{(3)}\left(c_{2}\right)\right)^{-1} \hat{B}_{\lambda}^{(4)}\left(c_{1}\right) G_{\lambda-1,0}^{\lambda-1}, \\
\tilde{M}_{\lambda}(0, v)=\left(-\hat{B}_{v-1}^{(3)}\left(c_{2}\right)\right)^{-1}\left[\hat{B}_{\lambda-v}^{(2)}(c) \tilde{M}_{\lambda}(0, v-1)\right. \\
\left.+\hat{B}_{\lambda}^{(4)}\left(c_{1}\right) G_{\lambda-1, v-1}^{\lambda-v}\right], \quad v=2,3, \ldots, \lambda, \\
\tilde{M}_{\lambda}(1,1)=-\left(\hat{B}_{0}^{(3)}\left(c_{2}\right)\right)^{-1} \hat{B}_{\lambda}^{(1)}(c) G_{\lambda-1,0}^{\lambda-1}, \tilde{M}_{\lambda}(1, v) \\
=-\left(\hat{B}_{v-1}^{(3)}\left(c_{2}\right)\right)^{-1}\left[\hat{B}_{\lambda-v+1}^{(2)}(c) \tilde{M}_{\lambda}(1, v-1)\right. \\
+\hat{B}_{\lambda-1}^{(3)}\left(c_{1}\right) \tilde{M}_{\lambda}(0, v-1)+\hat{B}_{\lambda-v+1}^{(2)}(c) G_{\lambda-1, v-1}^{\lambda-v} \\
\left.+\hat{B}_{\lambda-1}^{(4)}\left(c_{1}\right) G_{\lambda-2, v-1}^{\lambda-v+1}+\hat{B}_{v-1}^{(3)}\left(c_{2}\right) G_{\lambda-1, v-2}^{\lambda-v+1}\right]
\end{gathered}
$$

Заметим, что

$$
\begin{gathered}
c \frac{\partial}{\partial c} \hat{B}_{v}^{(i)}(c)=\hat{B}_{v}^{(i)}(c)\left(c \frac{\partial}{\partial c}-1\right), \\
c \frac{\partial}{\partial c}\left(\hat{B}_{v}^{(i)}(c)\right)^{-1}=\left(\hat{B}_{v}^{(i)}(c)\right)^{-1}\left(c \frac{\partial}{\partial c}+1\right) .
\end{gathered}
$$

Поэтому

$$
\begin{aligned}
& \left(2 T \frac{\partial}{\partial T}-c \frac{\partial}{\partial c}-c_{1} \frac{\partial}{\partial c_{1}}-c_{2} \frac{\partial}{\partial c_{2}}\right) G_{\lambda, 0}^{\lambda} \\
& =\frac{(-1)^{\lambda+1}}{(\lambda+1)}\left(\hat{B}_{\lambda-1}^{(2)}(c)\right)^{-1} \ldots\left(\hat{B}_{1}^{(2)}(c)\right)^{-1}\left(\hat{B}_{0}^{(2)}(c)\right)^{-1} \\
& \times\left(\hat{B}_{\lambda-1}^{(3)}\left(c_{1}\right)\right)^{-1}\left(\hat{B}_{0}^{(3)}\left(c_{2}\right) \ldots \hat{B}_{\lambda-2}^{(3)}\left(c_{2}\right) \hat{B}_{\lambda-1}^{(3)}\left(c_{2}\right)\right) \\
& \times\left(\hat{B}_{0}^{(2)}(c)\left(2 T \frac{\partial}{\partial T}-c \frac{\partial}{\partial c}-c_{1} \frac{\partial}{\partial c_{1}}-c_{2} \frac{\partial}{\partial c_{2}}\right) \tilde{M}_{\lambda}(1, \lambda)\right. \\
& +\hat{B}_{\lambda-1}^{(3)}\left(c_{1}\right)\left(2 T \frac{\partial}{\partial T}-c \frac{\partial}{\partial c}-c_{1} \frac{\partial}{\partial c_{1}}-c_{2} \frac{\partial}{\partial c_{2}}\right) \tilde{M}_{\lambda}(0, \lambda) \\
& \left.+\hat{B}_{\lambda}^{(4)}\left(c_{2}\right)\left(2 T \frac{\partial}{\partial T}-c \frac{\partial}{\partial c}-c_{1} \frac{\partial}{\partial c_{1}}-c_{2} \frac{\partial}{\partial c_{2}}\right) G_{\lambda-1, \lambda-1}^{0}\right) .
\end{aligned}
$$

Последнее слагаемое в круглых скобках равно нулю, поскольку ядра на слое $\lambda-1$ удовлетворяют соотношению (39). Первые два слагаемых обращаются в нуль в силу формул (42), (43). То есть (39) выполняется для $G_{\lambda, 0}^{\lambda}$.

Справедливость (39) для ядер вида $G_{\lambda, v}^{\lambda-v}, G_{\lambda-1, v}^{\lambda-v+1}$, $G_{\lambda-\tau, \tau}^{\lambda}, G_{\lambda-\tau, v}^{\lambda+\tau-v}$ можно доказать аналогично, используя формулы из [25].

Таким образом, свойство (39) выполняется для всех ядер на слое $\lambda$. Из эквивалентности (39) и (17) следует, что температурные соотношения выполняются для всех МЭ с любыми индексами $l, l_{1}, l_{2}$.

\section{Заключение}

Как было отмечено выше, существенной трудностью при реализации моментного метода является расчет матричных элементов интеграла столкновений. При сильном отклонении ФР от равновесия необходимо учитывать большое число членов разложения ФР по функциям Барнетта и, следовательно, использовать матричные элементы с большими индексами. В настоящей работе предложен алгоритм построения МЭ для произвольного потенциала взаимодействия между частицами с произвольным соотношением масс, при этом изотропные МЭ считаются известными. Подчеркнем, что рассматривается нелинейный интеграл столкновений, что дает возможность описывать эволюцию сильно неравновесных систем. Это может оказаться важным, например, при описании пороговых реакций. С целью проверки разработанной процедуры было проведено сравнение рассчитанных с ее помощью МЭ с МЭ, построенными раньше другим способом $[11,18]$ для модели твердых шаров, и получено совпадение результатов. В рассматриваемом алгоритме используются лишь скоростные рекуррентные соотношения, полученные в $[11,22]$. Они не 
зависят от закона взаимодействия частиц, являясь в этом смысле универсальными. Температурные рекуррентные соотношения для построенных МЭ оказываются тождествами, доказательству чего посвящен последний раздел работы. Алгоритм построения изотропных МЭ для произвольного потенциала взаимодействия с помощью $\Omega$-интегралов будет рассмотрен в нашей следующей работе.

Один из авторов (И.А. Эндер) благодарит РФФИ за частичную поддержку работы (грант № 15-08-03440).

\section{Список литературы}

[1] Burnett D. // Proc. London Math. Soc. 1935. Vol. 39. P. $385-430$.

[2] Burnett D. // Proc. London Math. Soc. 1935. Vol. 40. P. $382-435$

[3] Word C.A., Mintzer D. // Phys. Fluids. 1971. Vol. 14. N 3. P. 499-509.

[4] Kumar K. // Ann. Phys. 1966. Vol. 37. P. 113-141.

[5] Robson R.E., Ness K.F. // Phys. Rev. A. 1986. Vol. 33. P. 2068-2077.

[6] Ness K.F. // J. Phys. D: Appl. Phys. 1994. Vol. 27. P. $1848-1861$.

[7] White R.D., Ness K.F., Robson R.E. // Appl. Surf. Sci. 2002. Vol. 192. P. 26-49.

[8] Li B., Robson R.E., White R.D. // Phys. Rev. E. 2006. Vol. 74. P. 026405-13.

[9] Shizgal B.D., Dridi R. // Computer Phys. Commun. 2010. Vol. 181. P. 1633-1640

[10] Ender A.Ya., Ender I.A. // Phys. Fluids. 1999. Vol. 11. P. $2720-2730$.

[11] Эндер А.Я., Эндер И.А. Интеграл столкновений уравнения Больцмана и моментный метод. СПб., 2003. 224 с.

[12] Эндер А.Я., Эндер И.А., Герасименко А.Б. // ЖТФ. 2016. Т. 86. Вып. 11. С. 35-42.

[13] Mason E.A., Mc Daniel E.W. Transport Properties of Ions in Gases. NY:: Wiley, 1988. 489 p.

[14] Viehland L.A., Siems W.F. // J. Am. Soc. Mass. Spectrom. 2012. N 23. Vol. 11. P. 1841-1854.

[15] Чепмен C., Каулинг T. Математическая теория неоднородных газов. М.: Изд-во иностр. лит-ры, 1960. 510 с.

[16] Ферцигер Джс., Капер Г. Математическая теория процессов переноса в газах. М.: Мир, 1976. 557 с.

[17] Бакалейников Л.А., Тропп Э.А., Флегонтова Е.Ю., Эндер А.Я., Эндер И.А. ЖТФ. 2015. Vol. 85. Вып. 1. Р. 10-14.

[18] Ender A.Y., Ender I.A., Gerasimenko A.B. // Open Plasm. Phys. J. 2009. Vol. 2. P. 24-62.

[19] Эндер А.Я., Эндер И.А. // ЖТФ. 2003. Т. 83. Вып. 2. C. $6-12$.

[20] Hecke E. // Math. Ann. 1917. Vol. 78, P. 398-404.

[21] Hecke E. // Math. Zs. 1922. Vol. 12. P. 274-286.

[22] Ender A.Ya., Ender I.A. // Trans. Theor. Stat. Phys. 2007. Vol. 56. P. 563-588.

[23] Градштейн И.С., Рыжик И.М. Таблицы интегралов, сумм, рядов и произведений. М.: ФМ., 1962. 1097 с.

[24] Эндер А.Я., Эндер И.А., Бакалейников Л.А. // ДАН. 2011. Т. 437. Вып. 5. С. 621-623

[25] Бакалейников Л.А., Флегонтова Е.Ю., Эндер А.Я., Эндер И.А. // ЖТФ. 2016. Т. 86. Вып. 4. С. 10-20. 